\title{
The Characteristics of Magnetotelluric Responses for anisotropic conductivity
}

Bin Xiong, Guilin University of Technology; Hongzhu Cai*, University of Utah; Tianya Luo, Guilin University

of Technology

\section{Summary}

In this paper, we assume a $2 \mathrm{D}$ earth structure with anisotropic conductivity. One of the principal anisotropy axes is parallel to the striking direction and another axes is perpendicular to the surface of structure layer. By solving the variational problem with assigned boundary condition, we can compute the apparent resistivity for magnetotelluric (MT). Based on the forward modeling of MT field, we will analysis the difference of EM field for isotropic and anisotropic medium for $\mathrm{TE}_{\mathrm{Z}}$ polarization model. Our study shows that the MT field can be significantly affected by anisotropic medium and should be taken into consideration in many geophysical applications. We also analyzed the effect of anisotropic parameter (ration of horizontal conductivity to vertical conductivity) and dipping layer on MT field.

Keywords: finite element method; magnetotelluric; anisotropic medium; forward modeling

\section{Introduction}

We usually assume an istropic earth structure model in MT forward modeling. However, the conductivity property of rocks varies in many directions due to tempeture, pressue and some other physical and chemical effect (Shi, 1985). The anisotropic effect of conductivity will cause the electric field deviated from the direction of current. In oil and gas exploration, the anisotropic effect can sigificantly affect the evaluation of oil/gas saturation of rocks. The effect of anisotropy on MT method was first disscussed by Mann (1965). 2D MT forward modeling for anisotropic conductivity medium with finite element method was introduced by $\mathrm{Xu}$ and Zhao in 1985. In this paper, we compared the MT response of istropic and anisotropic medium for $\mathrm{TE}_{\mathrm{z}}$ polarization based on the study of $\mathrm{Xu}$ and Zhao. We analyzed the impact of anisotropic parameter on MT field.

2D boundary value problem for MT field in anisotropic medium

We considuer 2D geoelectric model in Cartesian coordinate system. In our study, we assue the postive $y$ axes directs upward; $\mathrm{x}$ and $\mathrm{z}$ axes are in transverse direction. The conductivity in transverse and longitudinal directions are denoted by $\sigma_{\|}$and $\sigma_{\perp}$. It is well known that the MT field can be seperated into $\mathrm{TM}_{\mathrm{z}}$ and $\mathrm{TE}_{\mathrm{z}}$ model (Allen and Susan, 2000). In this case, the $\mathrm{TM}_{\mathrm{z}}$ model with anisotropic conductivity shares the same partial differetial equations with the TMz model in isotropic medium with conductivity of $\sigma_{\|}$(Zhao and $\mathrm{Xu}, 1983$ ). In this paper, we will focus our dicussion on $\mathrm{TE}_{\mathrm{z}}$ model.

The MT problem for the model shows in figure 1 under the assumption of $\mathrm{TE}_{\mathrm{z}}$ polarization is described by the following equations (Xu, 1994):

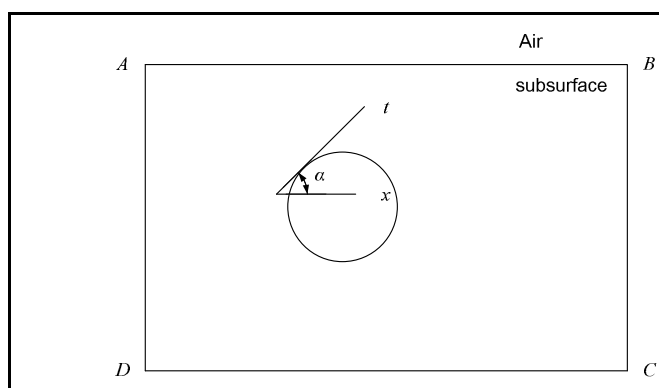

Figure 1: This Figure shows study domain for $\mathrm{TE}_{\mathrm{z}}$ model

$$
\begin{aligned}
& \nabla \cdot(\tau \nabla u(r))+\lambda u(r)=0, r \in \Omega, \\
& u(r)=1, \quad r \in A B, \\
& \frac{\partial u(\mathrm{r})}{\partial n}=0, r \in A D, B C \\
& \frac{\partial u(\mathrm{r})}{\partial n}+k u(\mathrm{r})=0, \quad \mathrm{r} \in C D
\end{aligned}
$$

where:

$$
u=H_{z}, \lambda=i \omega \mu, \tau=\left[\begin{array}{ll}
\tau_{11} & \tau_{12} \\
\tau_{21} & \tau_{22}
\end{array}\right]
$$

$$
\tau_{11}=\left(\frac{1}{\sigma_{\|}-i \omega \varepsilon} \sin ^{2} \alpha+\frac{1}{\sigma_{\perp}-i \omega \varepsilon} \cos ^{2} \alpha\right)
$$

$$
\tau_{22}=\left(\frac{1}{\sigma_{\|}-i \omega \varepsilon} \cos ^{2} \alpha+\frac{1}{\sigma_{\perp}-i \omega \varepsilon} \sin ^{2} \alpha\right)
$$$$
\tau_{12}=\tau_{21}=-\frac{1}{2}\left(\frac{1}{\sigma_{\|}-i \omega \varepsilon}-\frac{1}{\sigma_{\perp}-i \omega \varepsilon}\right) \sin (2 \alpha) .
$$

In the previous equations, $\nabla$ is the Hamilton operator for 2D problem, $\mathrm{k}=\sqrt{-\mathrm{i} \omega \mu \sigma_{\|}}, \Omega$ is our study domain, $\varepsilon$ is the dielectric constant, $\mu$ is the magnetic permeability, $i$ is the imaginary unit, $\omega$ is the angular frequency for 


\section{The Characteristics of Magnetotelluric Responses for anisotropic conductivity}

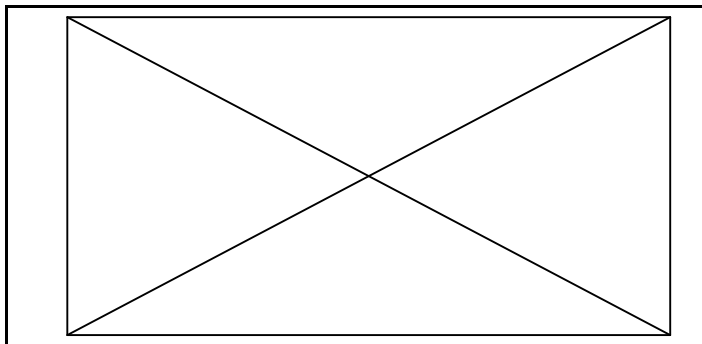

Figure 2: Four triangular element in a rectangular grid.

electromagnetic field and $\alpha$ is the angle between the physical property and the horizontal plane.

\section{Varitional problem for MT field in anisotropic medium}

The equivalent variational formulation for equation 1 to 4 is given as:

(9)

$$
\begin{gathered}
F(u)= \\
\iint_{\Omega}\left[\frac{1}{2} \nabla u \cdot(\tau \nabla u)-\frac{1}{2} \lambda u^{2}\right] d \Omega+\frac{1}{2} \int_{C D} \frac{k}{\sigma_{\|}-i \omega \varepsilon} u^{2} d \Gamma
\end{gathered}
$$

$$
\begin{gathered}
\left.u\right|_{A B}=1, \\
\delta F(u)=0
\end{gathered}
$$

The variational problem described from equation 9 to 11 can be solved numerically by using finite element method.

\section{Finite element method}

In our study, we discretized the $2 \mathrm{D}$ plane into a grid of rectangular grids and each rectangular cell is divided into four triangular elements by the two diagonal lines (Luo and Zhang 1987; Xiong and Luo, 2006) which is shown in figure 2 and figure 3 . For each triangular element, the linear interpolating function is given as follows (Lv, et al., 2011 ):

$$
u=N_{i} u_{i}+N_{j} u_{j}+N_{m} u_{m}
$$

where the subscripts $i, j, m$ are the nodes index for each triangular element in an anticlockwise manner. $N_{i}, N_{j}, N_{m}$ are the linear basis function.

In a discretized form, equation (9) can be written as follows:

$$
\begin{gathered}
F(u)=\sum_{\Omega} \iint_{e}\left[\frac{1}{2} \nabla u \cdot(\boldsymbol{\tau} \nabla u) \frac{1}{2} \lambda u^{2}\right] d \Omega_{e}+ \\
\sum_{C D} \int_{e_{C D}} \frac{1}{2} \frac{k}{\sigma_{\|}-i \omega \varepsilon} u^{2} d \Gamma_{e},
\end{gathered}
$$

We can compute each element's contribution to equation 13 and get a $4 \times 4$ local stiffiness matrix by eleminate the middle node. The local stiffiness matrix can be expanded to a global stiffiness matrix after assembling. The boundary value of field on $A B$ is substituted into the global equation to solve the field $H_{z}$ in each node. The apparent resistivity on the earth's surface can be calculated from the field.

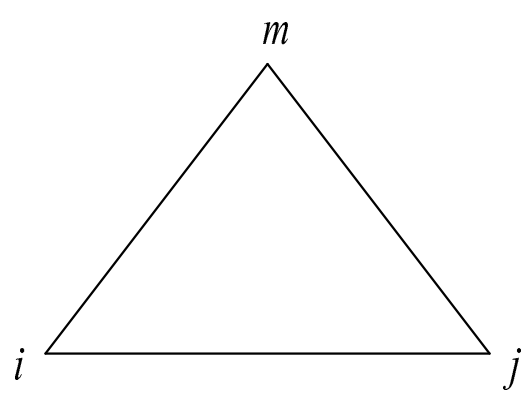

Figure 3: A triangular element with nodes index.

\section{Analysis of forward modeling result}

Model 1: syncline conductive body

Figure 4 shows an illustration of this model. For this model we considered both isotropic conductivity and anisotropic conductivity. The isotropic conductivity is given as the geometric mean of anisotropic conductivities ( $\rho=$ $\left.\sqrt{\rho_{\|} \cdot \rho_{\perp}}\right)$. By taking this value, we can prove that the different MT response for the isotropic and anisotropic model is mainly caused by the perturbation of physical properties in different direction rather the value of resistivity. Figure 5 shows the numerical result for different frequency. As we can see that for the first frequency, the MT response for isotropic and anisotropic medium are 24 times difference. Therefore, we find an necessity to consider anisotropic effect in the processing and interpretation of MT data. As the frequency increase, the anisotropic effect will decrease monotonically. 


\section{The Characteristics of Magnetotelluric Responses for anisotropic conductivity}

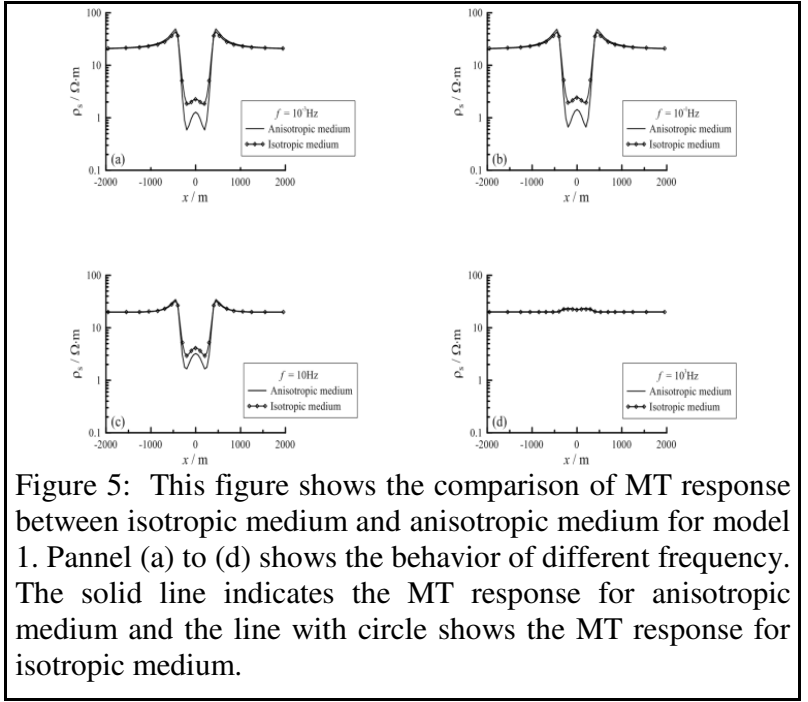

Model 2 : dipping conductive body

The modle is shown in figure 6 with different values for $\alpha$ to be $0^{\circ}, 45^{\circ}$ and $90^{\circ}$. Figure 7 shows the change of apparent resistivity for different $\alpha$ for $\mathrm{TE}_{\mathrm{z}}$ polarization. As we can see that there is some artificial anomaly around our conductive body with resistivity higher than the wall-rock. As the angle $\alpha$ increase, the artificial anomaly starts to diminish.



\section{Model 3: Rectangular model}

In this model, we assume that the background resistivity is $\rho_{2}=1000 \Omega \cdot m$. A rectangular body is embeded in this

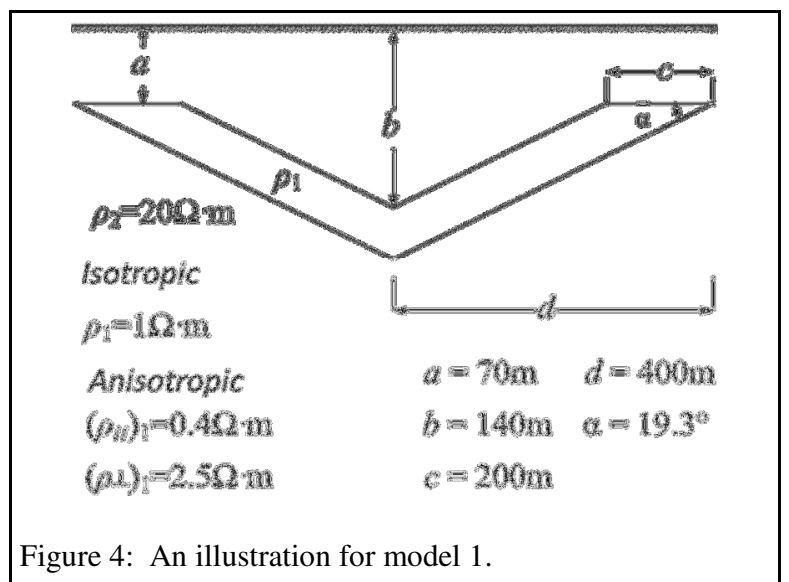

background. Figure 8 is an illustration of this model. For this model, we have two submodels. For submodel a, we fix the horizontal reistivity of the anomalous body and change the vertical resistivity from $10 \Omega \cdot m$ to $10^{6} \Omega \cdot m$. For submodel b, we fix the vertical resistivity of the anomalous body and change the horizontal resistivity in a simimar manner as submodel a. The parameters of these two submodels are shown in table 1 .

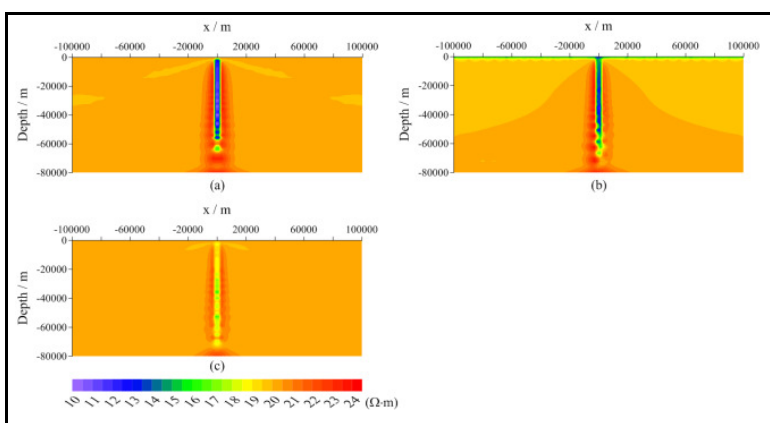

Figure 7: Apparent resistivity calculated for model 2. (a) $\alpha=0^{\circ}$, (b) $\alpha=45^{\circ}$, (c) $\alpha=90^{\circ}$

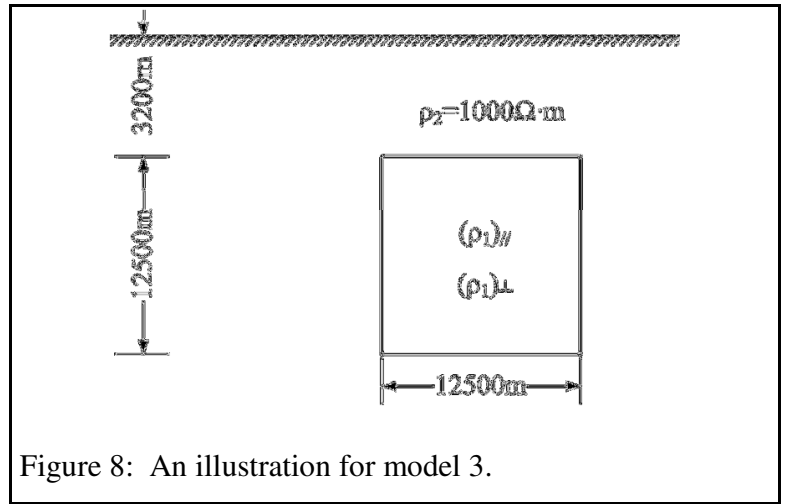




\section{The Characteristics of Magnetotelluric Responses for anisotropic conductivity}

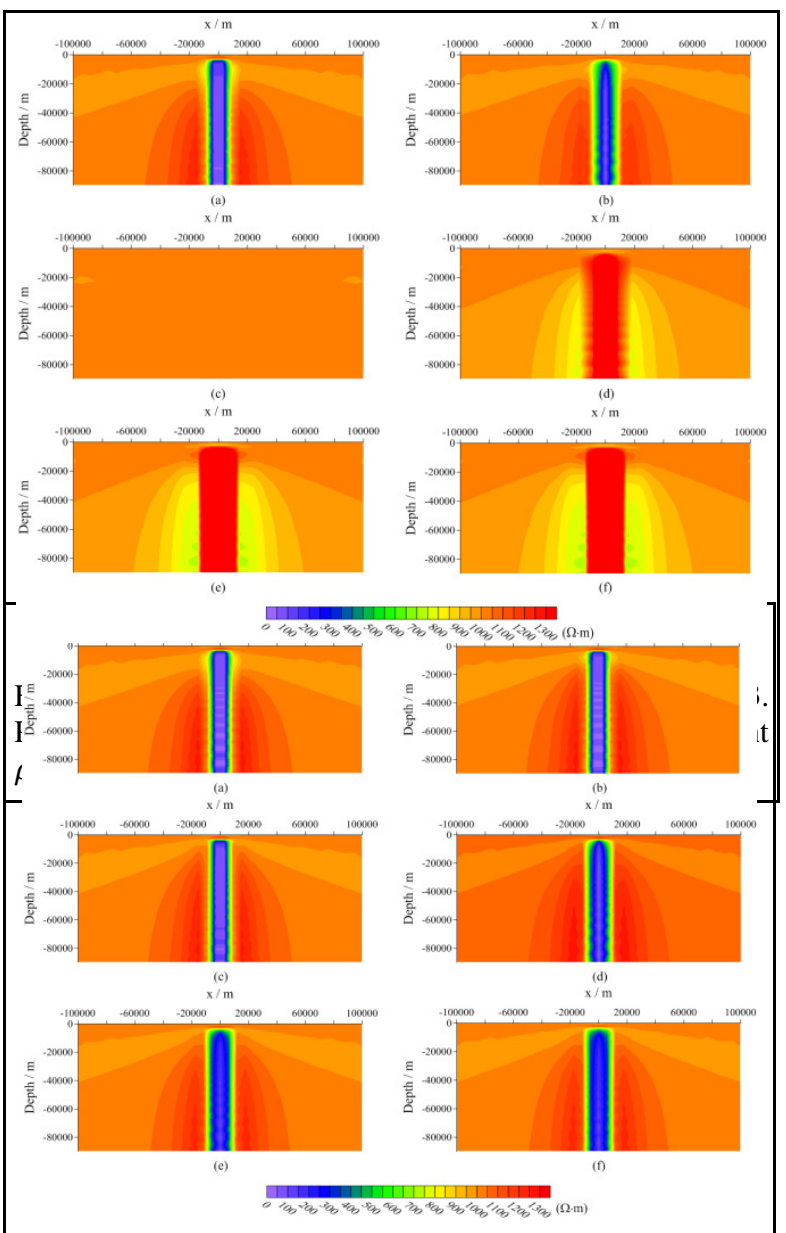

Figure 9: Apparent resistivity for submodel a in model 3. Pannel (a) to (e) shows the result for different $\rho_{\mathbf{1}}$.

\begin{tabular}{|c|c|c|c|c|c|c|c|}
\hline Submodel & \multicolumn{7}{|c|}{ Resistivity $(\Omega \cdot \mathrm{m})$} \\
\hline \multirow{2}{*}{$a$} & $\rho_{1 \|}$ & \multicolumn{6}{|c|}{$\rho_{1 \perp}$} \\
\cline { 2 - 8 } & 1 & 10 & $10^{2}$ & $10^{3}$ & $10^{4}$ & $10^{5}$ & $10^{6}$ \\
\hline \multirow{2}{*}{$b$} & $\rho_{1 \perp}$ & \multicolumn{6}{|c|}{$\rho_{1 \|}$} \\
\cline { 2 - 8 } & 1 & 10 & $10^{2}$ & $10^{3}$ & $10^{4}$ & $10^{5}$ & $10^{6}$ \\
\hline
\end{tabular}

Table 1 . Resistivity parameter for model 3.

The apparent resistivity for submodel $a$ and submodel $b$ are shown in figure 9 and figure 10 . From figure 9 , we can see that if we fix the horizontal resistivity and increase the vertical resistivity, the apparent resistivity always shows a low resistivity anomaly compared to the background (wallrock resistivity) even if the anomalous resistivity is higer than the wall-rock resistivity in our model.
For figure 10, the vertical resistivity of the anomalous body is fixed and we will increase the horizontal anomalous resistivity from a value lower than the wall-rock resistivity to another value larger than the wall-rock resistivity. We can identify the low resistivity anomaly, compared to the wall-rock, from the apparent resistivity figure if the horizontal resistivity of the body is lower than the wallrock resistivity in our model. If the horizontal resistivity of the body is higer than the wall rock in our model, we are able to identify the higher resistivity anomaly from the apparent resistivity figure. However, if the horizontal resistivity of the the anomalous body is equal to the wallrock resistivity, we will not be able to find the anomlous body from the apparent resistivity figure. In an anisotropic medium, the horizontal resisitivity has a larger impact on the effective resistivity than the vertical resistivity for our model study.

\section{Conclusion}

In this study, we compared the MT response for a isotropic medium and an anisotropic medium. We also studied the impact of anisotropic parameter on MT method. Based on our study, we find that the anisotropic effect cannot be simply negelect in practical application MT method especially for low frequency.

We also analyzed the effect of the dipping layer angle to the MT response for an anisotropic medium. As we can see from our model study, the horizontal dimension of the low resistivity body in the apparent resistivity figure decrease as the dipping angle increase. The area nearby the anomalous body in the apparent resistivity figure is charactized by some artificial anomaly with the resistivity higher than the wall-rock reistivity and this effect is more serious for small dipping angle.

In an anisotropic medium, the MT response is primiarily affected by the horizontal resistivity than the vertical resistivity.

\section{Acknowledgements}

The authors acknowledge the support of the University of Utah's Consortium for Electromagnetic Modeling and Inversion (CEMI), Guilin Uiversity of Technology. 
http://dx.doi.org/10.1190/segam2013-0438.1

\section{EDITED REFERENCES}

Note: This reference list is a copy-edited version of the reference list submitted by the author. Reference lists for the 2013 SEG Technical Program Expanded Abstracts have been copy edited so that references provided with the online metadata for each paper will achieve a high degree of linking to cited sources that appear on the Web.

\section{REFERENCES}

Taflove, A., and S. Hagness, 2000, Computational electrodynamics: The finite-difference time-domain method (second edition): Artech House, 70-72.

Lv, Y. Z., B. Xiong, and T. X. Xue, 2011, Fundamentals of geophysical data processing: Geology Press, 46-47 (in Chinese).

Luo, Y. Z., and G. Q. Zhang, 1987, Application of electronic s in electrical prospecting: Wuhan College of Geology Press, 69-83 (in Chinese).

Maan, J. E., Jr., 1965, The importance of anisotropic conductivity in magnetotelluric interpretation: Journal of Geophysical Research, 70, 2940-2942, http://dx.doi.org/10.1029/JZ070i012p02940.

Shen, J. S, and N. C. Guo, 2008, Study on the apparent resistivity and magnetic field responses of a layered earth with arbitrary anisotropy: Chinese Journal of Geophysics, 51, 1608-1619.

Shi, Y. J., G. D. Liu, and G. Y. Wu, 1985, Course of magnetotelluric sounding: Seismological Press, 2021 (in Chinese).

Xiong, B., and Y. Z. Luo, 2006, Finite element modeling of 2.5-D TEM with block homogeneous conductivity: Chinese Journal of Geophysics, 49, 515-525, http://dx.doi.org/10.1002/cjg2.862.

Xu, S. Z., 1994, FEM in geophysics: Science Press, 241-145 (in Chinese).

Xu, S. Z., and S. K. Zhao, 1985, Solution of magnetotelluric field equations for a two-dimensional anisotropic geoelectric section by the finite element method: Acta Seismologica Sinica, 7, 80-90.

Zhao, S. K., and S. Z. Xu, 1983, The calculation of good conductor inserted in 2-D model to obtain magnetotelluric sounding curves by FEM: Computing Techniques for Geophysical and Geochemical Exploration, 1, 14-21. 